\title{
"Unseen" Caregivers: The disproportionate gender balance and role of females in the home-based care of lymphatic filariasis patients in Malawi
}

\author{
Martindale $\mathrm{S}^{1 *}$, Mackenzie $\mathrm{C}^{1,2}$, Mkwanda $\mathrm{S}^{3}$, Smith $\mathrm{E}^{1}$, Stanton $\mathbf{M}^{1}$, Molyneux $\mathrm{D}^{1}$, and Kelly-Hope $\mathrm{L}^{1}$ \\ ${ }^{1}$ Liverpool School of Tropical Medicine, Pembroke Place, UK \\ ${ }^{2}$ Michigan State University, East Lansing, MI 48824, USA \\ ${ }^{3}$ Ministry of Health, Capital Hill, Lilongwe, Malawi
}

\begin{abstract}
Objective: This study examines the gender of home-based caregivers for people affected by lymphatic filariasis (LF) lymphoedema.

Methods: In total, 69 LF lymphoedema cases in Malawi were questioned about the assistance they received with a focus on the gender of the caregiver and the type of support provided.

Results: Of the 35 cases who required daily assistance, 27 indicated the gender of the caregiver, of which 20 were female (74.1\%), and most commonly daughters, sisters or school-aged girls. This care was usually only provided during episodes of painful disabling acute-dermatolymphangioadenitis (ADLA) attacks. The males who provided care were most commonly husbands.
\end{abstract}

Conclusion: The role of female caregivers is 'unseen' and this has considerable domestic, educational and economic implications. This gender imbalance also poses barriers to Goal 5 of the Sustainable Development Goals, specifically Target 5.4 that aims to recognize and value unpaid care and domestic work.

\section{Introduction}

Lymphatic Filariasis (LF) is a disabling and disfiguring parasitic disease, and one of 17 Neglected Tropical Diseases (NTDs) defined by the World Health Organization (WHO), which affect the poorest and most marginalised communities in the world [1]. Globally, recent figures estimate that 36 million people suffer from the clinical manifestations of LF, specifically limb lymphoedema (16.68 million) and hydrocele in men (19.43 million), which accounts for at least 2.8 million disability-adjusted life years (DALYs) [1,2]. These disabling and disfiguring conditions require specific morbidity management and disability prevention (MMDP) measures, as they inflict a complex range of physical, social, psychological and economic hardships on those affected.

These clinical-related hardships are likely to extend beyond the patient to their caregivers at home, however, there is little information on caregivers, apart from the recent estimates on the burden of depression amongst caregivers of LF patients and an analysis of focus group discussions with LF patients, families, community members and programme volunteers in India [3-5]. The role of caregivers appears to be largely unrecognized, or 'unseen', and could be a significant determining factor in their own ability to work and provide for their family. A further concern is the role of child caregivers, as they may have to make a choice between personal development (such as obtaining an education) and caring for a disabled relative.

A previous study conducted by the authors in Malawi [6] reported that a diversity of different family members provide care to LF patients in endemic areas of the Shire Valley in the Southern region of the country; especially during episodes of painful, disabling acutedermatolymphangioadenitis (ADLA) attacks. The gender of the caregiver was not specifically examined previously as it was anecdotal information and not the focus of the original study by Martindale et al. [6]. However, as it is likely that women and girls play a significant role, it is important to consider gender in understanding the role of caregivers in providing essential support and services to LF patients. Caregivers are important contributors to the WHO MMDP goals for LF, and are an important factor in achieving the Sustainable Development Goals (SDG); specifically Goal 5 that aims to achieve gender equality and empower all women and girls through specific targets (https://sustainabledevelopment.un.org/sdgs). Target 5.4 of the SDG is especially relevant as it aims to recognize and value unpaid care and domestic work.

To begin to address this neglected area of caregivers, their role in MMDP service provision for LF patients, and the potential impact on female family members; the descriptive interview data collected previously was re-examined to determine if there were any trends in the gender of the caregivers for LF patients in Malawi [6].

Correspondence to: Sarah Martindale, Liverpool School of Tropical Medicine, Pembroke Place, UK, Tel: +44151 705 3774; E-mail: sarah.martindale@lstmed.ac.uk

Key words: caregivers, equity, gender, lymphatic filariasis, neglected tropical diseases, sustainable development goals

Received: February 14, 2017; Accepted: March 07, 2017; Published: March 09, 2017 


\section{Methods}

Interviews and descriptive data was collected during a patient survey conducted in Chikwawa District, Malawi in 2013 as previously reported [6]. The survey assessed 69 lymphoedema cases and the impact of their clinical condition of eight main 'domains' of their lives, including mobility, self-care, usual activities, pain, cognition, anxiety, social participation and economic impact. The original analysis found that people affected required help with their 'self-care' and 'usual activities' of daily living, including information on i) whether they required assistance ii) with what activities do they need assistance and iii) how many people were involved. The re-analysis of the collected data focused specifically on the details of personnel who provided the care and assistance.

\section{Results}

For the 'self-care' domain, approximately half of participants (35/69; $50.7 \%$ ) stated they needed some form of assistance or care, mainly when they were having an ADLA attack. Of those, 27 participants indicated the gender of the caregiver, and of these $20(74.1 \%)$ were female, six (22.2\%) were male and one person (3.7\%) had assistance from both a male and female (Figure 1A). The majority of the female caregivers were daughters $(9 / 20 ; 45 \%)$ with smaller proportions recorded to be wives $(6 / 20 ; 30 \%)$, sisters $(4 / 20 ; 20 \%)$, and in one instance was a young girl from a neighboring village $(1 / 20 ; 5 \%)$ (Figure $1 \mathrm{~B})$. The majority of male caregivers were husbands $(4 / 6 ; 66.7 \%)$, with one being a son $(1 / 6 ; 16.65 \%)$ and another a young boy in the village $(1 / 6 ; 16.65 \%)$. All caregivers assisted patients with a range of needs during an ADLA attack, with five patients specifically stating that they received assistance with washing/bathing from a female caregiver.

For the 'usual activity' domain, a smaller number of participants $(13 / 69 ; 18.8 \%)$, stated that they needed assistance or care, mainly during an ADLA attack. Seven participants stated the gender of the caregiver, and of these, five (38.5\%) were female, and two (15.4\%) were male (Figure 1C). All caregivers assisted the LF patients during an ADLA attack, and of the female caregivers, two were daughters (assisted with childcare, housework), two were wives and one was a lady from the village (Figure 1D). The two male caregivers were husbands. All caregivers assisted during an ADLA attack; such care was identified as childcare, general housework, drawing and carrying water, washing and cooking.

A. Caregiver to self-care
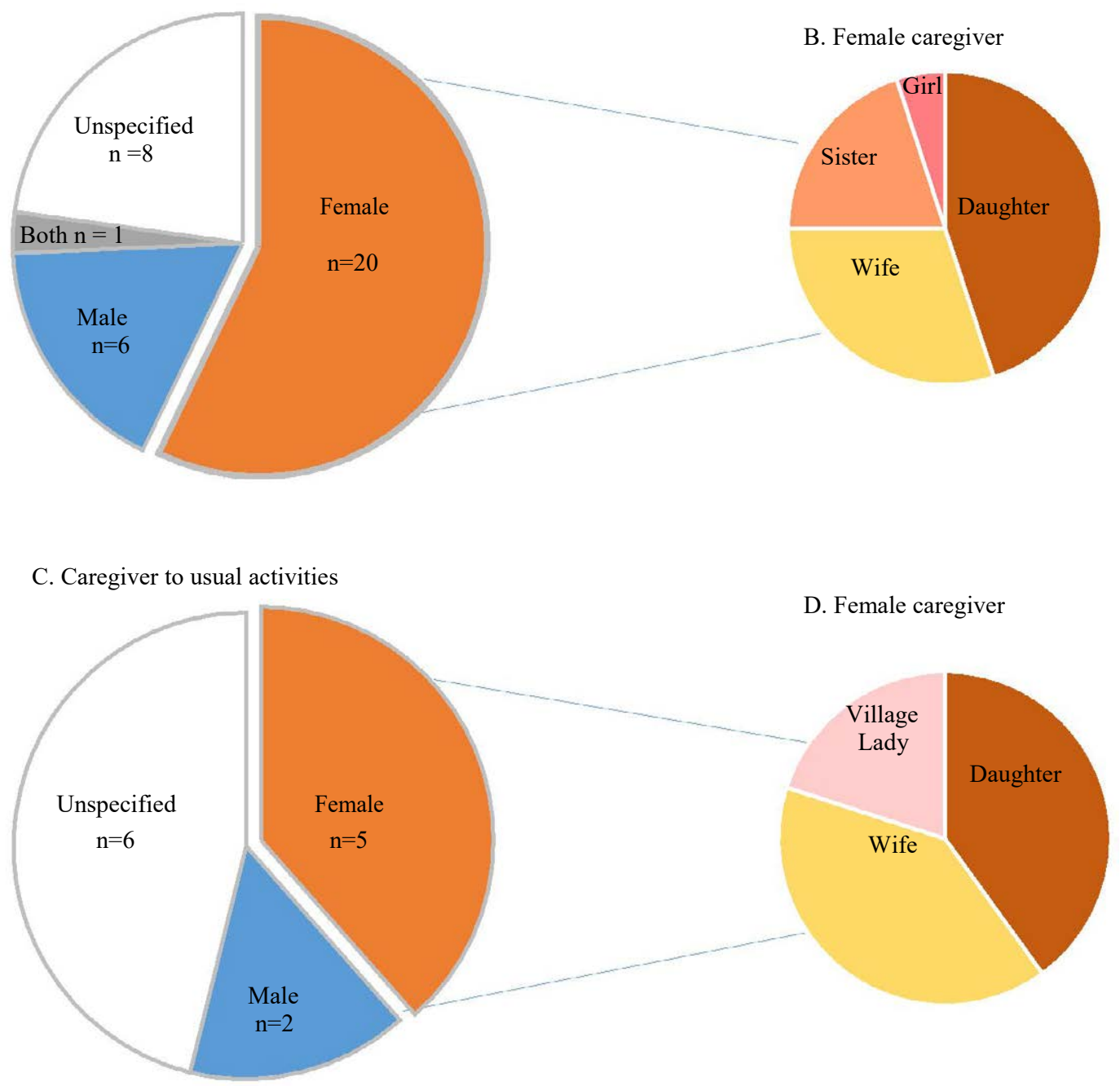

Figure 1. Gender of home caregivers for people affected by lymphoedema 


\section{Conclusion}

This study adds to the findings of Martindale et al. [6], that filarial lymphoedema causes significant hardship not only to the patient, but in many cases also to those providing care to the patient. Although the numbers presented in the study and analysis are small, they provide insights into the 'unseen' burden of caregivers, especially those who assist LF patients during painful ADLA attacks. These attacks are extremely debilitating to patients, can occur frequently and, often in severe cases last more than a week [6]. Understanding the scale of the burden of caregivers, and specifically on females, will require more directed studies to highlight those who may be missing out on an education or employment opportunities. Ton et al. [3] estimated that five million LF patients worldwide will suffer severely enough to require a caregiver, of which a significant majority are likely to be women and girls. Addressing such gender inequalities will be crucial in making progress towards SDG5, and in recognising and valuing unpaid care and domestic work. Such a focus will also help to recognise the contribution of women and girls to the WHO MMDP goals for LF, as well as helping to provide public services, infrastructure and social protection policies and the promotion of shared responsibility within the household and the family, as nationally appropriate, as set out in SDG Target 5.4. In summary, helping to ensure no-one is left behind.

\section{Acknowledgments}

The authors acknowledge the research team in Malawi and the patients themselves for agreeing to share their information on such a sensitive and personal matter.

\section{Funding information}

This work was supported by grants from the UK Department for International Development (DFID) to the Centre of Neglected Tropical Diseases, Liverpool School of Tropical Medicine for the elimination of lymphatic filariasis as a public health problem.

\section{Ethical approval}

Ethical approval for this study was obtained from the Research Ethics Committee at the Liverpool School of Tropical Medicine, UK; and the National Health Sciences Research Committee at the Ministry of Health, Malawi.

\section{References}

1. World Health Organization. Lymphatic filariasis. World Health Organization, Geneva

2. Ramaiah KD, Ottesen EA (2014) Progress and impact of 13 years of the global programme to eliminate lymphatic filariasis on reducing the burden of filarial disease. PLoS Negl Trop Dis 8: e3319. [Crossref]

3. Ton TG, Mackenzie C, Molyneux DH (2015) The burden of mental health in lymphatic filariasis. Infect Dis Poverty 4: 34. [Crossref]

4. Litt E, Baker MC, Molyneux D (2012) Neglected tropical diseases and mental health: a perspective on comorbidity. Trends Parasitol 28: 195-201. [Crossref]

5. Cassidy T, Worrell CM, Little K, Prakash A, Patra I, et al. (2016). Experiences of a Community-Based Lymphedema Management Program for Lymphatic Filariasis in Odisha State, India: An Analysis of Focus Group Discussions with Patients, Families, Community Members and Program Volunteers. PLoS Negl Trop Dis. 10 (2), e0004424.

6. Martindale S, Mkwanda SZ, Smith E, Molyneux D, Stanton MC, et al. (2014) Quantifying the physical and socio-economic burden of filarial lymphoedema in Chikwawa District, Malawi. Trans R Soc Trop Med Hyg 108: 759-767. [Crossref]

Copyright: (C2017 Martindale S. This is an open-access article distributed under the terms of the Creative Commons Attribution License, which permits unrestricted use, distribution, and reproduction in any medium, provided the original author and source are credited. 